\title{
Le système pronominal du français louisianais
}

\author{
Francine Girard Lomheim (Université d'Agder, Norvège)
}

\begin{abstract}
This paper discusses the pronominal system of Louisiana French, a variety of French spoken mainly in the south-western part of Louisiana State. The analysis of data (Girard Lomheim, 2016) shows that although Louisiana French clitics share certain features with clitics from other informal varieties of French and dialects of French, they cannot be analysed along quite the same lines. They have reached a more advanced stage of grammaticalization. They are moving away from the status of syntactic argument towards the status of agreement marker. The fact that they are subject to strong phonetic erosion and have been gradually replaced by weak pronouns shows that they are coming closer to the ultimate stadium of their grammaticalization cycle (van Gelderen, 2011), the null stadium.

The progressive reduction of the clitic paradigm is accompanied by the emergence of a new class of weak pronouns, which leads us to claim that the pronominal system of this vernacular is organized in three classes of pronouns: strong pronouns and two classes of deficient pronouns: weak pronouns and clitics in the terms of Cardinaletti et Starke (1994 and 1999). We claim that the difference between strong and weak pronouns should be addressed in terms of syntax and morphology whereas the difference between weak pronouns and clitics should be accounted for in terms of morphosyntax and phonology.
\end{abstract}

Keywords: variation, language change, clitics, weak pronouns, deficiency

\section{Introduction}

Cet article a pour objectif de présenter les paradigmes des pronoms personnels du français louisianais. Des analyses préliminaires de ce système ont été exposées dans plusieurs articles (Girard, 2012, 2013et 2014) et c'est l'analyse définitive à laquelle est parvenue ma recherche sur cette variété de français exportée (Girard Lomheim : 2017) qui sera présentée ici.

Les clitiques du français sont généralement analysés selon deux axes, l'un pour le français standard (FS), et l'autre pour les français parlés informels (FPI) à l'intérieur et à l'extérieur de l'Hexagone. Le français louisianais étant un français essentiellement oral, qui s'est développé avec des contacts sporadiques avec le français de France pendant près de trois cents ans, on est en droit de penser que ses clitiques auront plus de traits en commun avec ceux des FPI qu'avec ceux du FS. Je montrerai que, si c'est effectivement le cas, ils s'en distinguent aussi par certains aspects qui font de ce français une variété originale. 


\section{Corpus}

Les données qui sous-tendent ce travail proviennent principalement du corpus recueilli en 1997 sous la direction du Dr Sylvie Dubois, professeure à la Louisiana State University (LSU) à Bâton-Rouge en Louisiane. Ce corpus est composé d'interviews réalisées par des locuteurs natifs, originaires de la même communauté que les locuteurs interviewés. Pour cette étude, 30 locuteurs originaires de la région autour de Lafayette ont été sélectionnés : 15 au nord, dans la communauté de Eunice, située dans la paroisse de St Landry, et 15 au sud, dans la communauté d'Abbeville, située dans la paroisse de Vermilion. La carte qui suit montre la situation centrale de ces communautés, localisées au cœur du triangle francophone louisianais, dénommé aussi Acadiane.

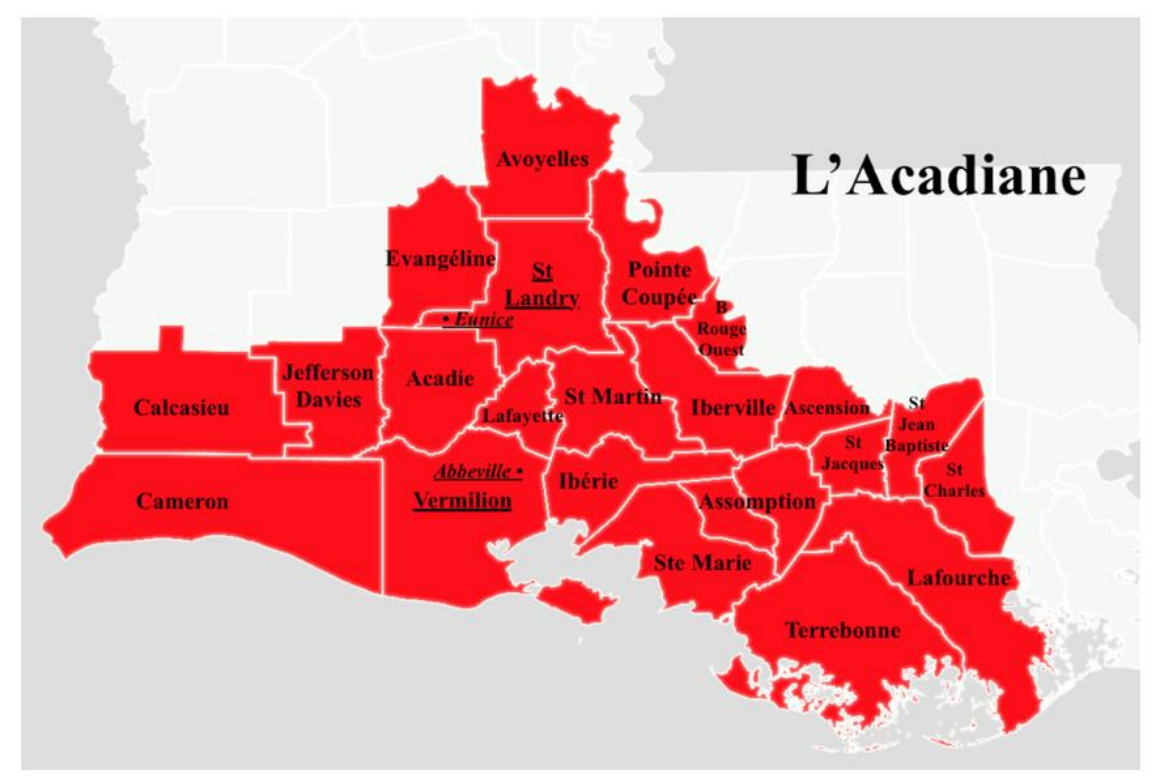

Ces 30 locuteurs se répartissent sur les trois tranches d'âge traditionnelles, les aînés (60 ans et plus), les cadets (40-59 ans) et les jeunes (19-39 ans). L'objectif de cette recherche étant de donner une description du système tel qu'il est parlé par des locuteurs performants, le degré d'aptitude langagière a également été pris en compte et les semi-locuteurs ont été exclus.

\section{Analyse des clitiques du français}

Ainsi que mentionné dans l'introduction, deux approches prédominent en ce qui concerne les clitiques du français, l'une pour le FS et l'autre pour les FPI. Analyse

\subsection{Français standard}

Les analyses proposées pour le FS relèvent de l'approche dite syntaxique. Les analyses de Kayne $(1975,1983)$, bien qu'anciennes, demeurent actuelles encore aujourd'hui. Il s'agit d'une analyse par mouvement. Kayne considère que les pronoms sujets conjoints, je, tu, il, elle, on, nous, vous, ils, elles ainsi que les pronoms objets conjoints me, te, le, la, lui, se, nous, vous, les et leur, sont générés à la base dans la position argumentale du sujet, la position du DP plein correspondant, et qu'ils montent ensuite se placer, en structure de surface, dans 
une position adjointe au verbe tête par le biais d'une règle baptisée placement du clitique (CLPL) (Kayne, 1975). Les pronoms clitiques du FS sont donc analysés comme des arguments dotés d'un rôle-thêta, c'est-à-dire correspondant aux arguments du verbe.

\subsubsection{Les clitiques sujets}

Pour ce qui est des clitiques sujets, Kayne opère avec deux analyses distinctes pour les clitiques qui apparaissent en position préverbale, les proclitiques, et ceux qui apparaissent en position postverbale, les enclitiques. Les proclitiques sujets ont le statut d'argument car ils fonctionnent comme de véritables sujets, au même titre que les DP sujets. Le proclitique est, selon lui, un argument pronominalisé puis déplacé de sa position profonde de sujet vers une position adjointe au verbe. Il est ensuite cliticisé, en phonologie, à l'issue d'une règle phonologique tardive de caractère postsyntaxique. Une phrase comme Il arrive, aura la structure arborescente ci-après où $t$ est la trace (ou copie) du sujet déplacé $I l$ :

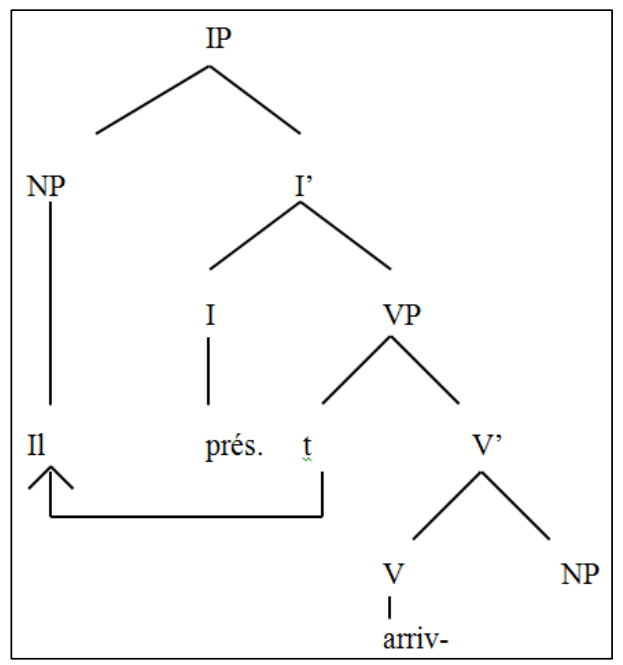

La syntaxe des enclitiques sujets, se distingue de celle des proclitiques essentiellement par deux points : l'enclitique peut apparaître en cooccurrence avec le SN sujet et l'enclitique ne peut pas s'ellipser, ce qui mène Kayne (1983) à les analyser non comme des arguments, mais des clitiques dès la structure profonde, et à argumenter en faveur d'une cliticisation syntaxique des enclitiques.

\subsubsection{Les clitiques objets}

En ce qui concerne les pronoms clitiques objets du FS, ils sont - tout comme les proclitiques sujets - en distribution complémentaire avec les DP pleins et les PP correspondant. Kayne les analyse en conséquence comme des arguments générés dans la même position que les DP et les PP dans la structure profonde avant d'être ensuite déplacés dans leur position de surface où ils sont cliticisés au verbe tête.

\subsection{Les français parlés informels (FPI)}

L'approche postulée pour les proclitiques des français parlés dits informels (FPI) est une approche de type dite morphologique. La dérivation des clitiques est une dérivation 
directe dans la structure de base sur les lignes de celle prônée par Rizzi (1986) pour l'italien dialectal. Cette approche est, entre autres, celle proposée par Roberge (1990) pour le français québécois et le français pied-noir, Zribi-Hertz (1994), pour le français parlé dans l'Hexagone, et Auger (1995 et 2003), pour le français québécois et le picard, respectivement.

Tous argumentent en faveur du statut non argumental des clitiques sujets dans ces variétés et les traitent comme des affixes marqueurs d'accord. Leurs analyses s'appuient essentiellement sur les faits suivants : le caractère obligatoire du sujet clitique devant le verbe et le redoublement du sujet par un clitique. Selon eux, le fait que les FPI acceptent la cooccurrence d'un sujet clitique et d'un DP plein sujet indique que les clitiques pronominaux de ces variétés doivent être analysés non comme des arguments, mais comme des affixes marqueurs d'accord, le verbe ne pouvant accepter deux arguments sujets. En FPI, la cliticisation n'est donc pas phonologique, ainsi que Kayne (1975) le suggère pour les proclitiques du FS, mais morphosyntaxique. La phrase Il mange se verra assignée la structure arborescente suivante :

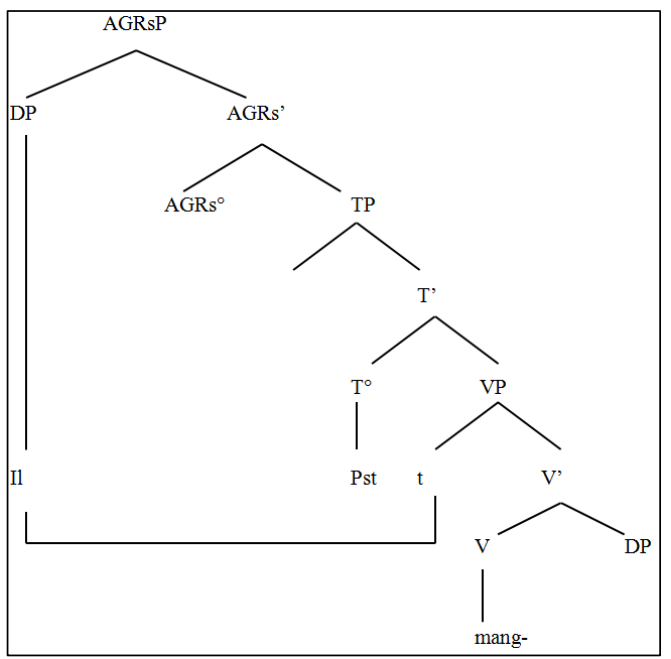

Dans le cas des clitiques objets, ils sont le plus souvent analysés comme des affixes eux aussi bien que cette analyse se voie fréquemment remise en question car le redoublement de l'objet n'est pas attesté dans ces français informels.

\section{Analyse des clitiques du français louisianais}

Si nous nous tournons maintenant vers les pronoms du français louisianais, nous pouvons constater qu'ils présentent, comme on était en droit de s'y attendre, plusieurs points communs avec ceux des FPI et que l'approche morphologique est celle qui rend le mieux compte de leurs caractéristiques.

\subsection{Les formes}

L'étude du parler des 30 locuteurs louisianais sélectionnés permet d'établir, dans un premier temps, le tableau suivant : 


\begin{tabular}{|c|c|c|c|c|c|c|}
\hline & & \multicolumn{4}{|c|}{ Formes conjointes } & \multirow[b]{2}{*}{$\begin{array}{l}\text { Formes } \\
\text { disjointes }\end{array}$} \\
\hline Nombre & Personne & Sujet & $\begin{array}{l}\text { Objet } \\
\text { direct }\end{array}$ & $\begin{array}{c}\text { Objet } \\
\text { indirect } \\
\text { datif }\end{array}$ & $\begin{array}{c}\text { Objet } \\
\text { indirect }\end{array}$ & \\
\hline \multirow{5}{*}{ Singulier } & $1^{\text {re }}$ & je & \multicolumn{2}{|c|}{ me, moi } & & moi \\
\hline & $2^{\mathrm{e}}$ & $\begin{array}{c}\text { tu, ça, vous (pl. } \\
\text { de politesse) }\end{array}$ & \multicolumn{2}{|c|}{$\begin{array}{l}\text { te, toi, vous (pl. de } \\
\text { politesse) }\end{array}$} & & $\begin{array}{l}\text { toi, vous- } \\
\text { autres }\end{array}$ \\
\hline & $3^{\mathrm{e}} \mathrm{m}$ & il, ça & \multirow{2}{*}{\multicolumn{2}{|c|}{ la, y, lui (rare), se }} & en, y & lui, ça \\
\hline & $3^{\mathrm{e}} \mathrm{f}$ & alle, ça & & & en, $y$ & elle, ça \\
\hline & $\begin{array}{l}3^{\mathrm{e}} \text { imp., } \\
\text { neutre, } \\
\text { indéfini }\end{array}$ & ça, ce, il, on & le (rare) & \multicolumn{2}{|c|}{ lé } & ça \\
\hline \multirow{3}{*}{ Pluriel } & $1^{\text {re }}$ & on, nous-autres & \multicolumn{2}{|c|}{ nous, se } & & nous-autres \\
\hline & $2^{\mathrm{e}}$ & vous-autres, ça & \multicolumn{2}{|c|}{ vous, se } & & vous-autres \\
\hline & $3^{\mathrm{e}}$ & $\begin{array}{l}\text { ils, ça, eusse, } \\
\text { eux-autres }\end{array}$ & \multicolumn{2}{|c|}{ les, lé, leur (rare), se } & en, $y$ & $\begin{array}{c}\text { eux-autres } \\
\text { eusse, ça }\end{array}$ \\
\hline
\end{tabular}

Un regard sur les paradigmes permet de constater plusieurs écarts qui seront commentés dans les sections qui suivent.

\subsection{Le paradigme disjoint}

Le paradigme disjoint se caractérise par l'emploi des formes nous-autres, vous-autres, eux-autres et eusse qui ont évincé les formes nous, vous et eux. Nous noterons aussi que la $3 \mathrm{e}$ personne du pluriel n'est pas marquée pour le genre et que le pronom elles est absent du paradigme. Pour ce qui est de leur fonctionnement, ils arborent un comportement similaire à ceux des autres variétés de français, à savoir qu'ils fonctionnent comme des syntagmes déterminatifs, des DP pleins, des catégories maximales. Ils sont générés dans la position de spécifieur de VP dans la structure de base, position argumentale du sujet, où le verbe leur assigne un rôle-thêta ; ils sont ensuite déplacés de cette position pour venir s'attacher à une position de spécifieur, Spec IP.

\subsection{Les paradigmes conjoints}

\subsubsection{Les formes}

L'examen des paradigmes des formes conjointes sujets permet de remarquer tout d'abord l'expansion du champ d'emploi de pronom ça et dévoile l'absence de plusieurs pronoms qui ont cédé la place à d'autres pronoms. Ainsi nous est remplacé par on et par la forme composée nous-autres; le vous du pluriel fait place à la forme composée vous-autres. Pour ce qui est des troisièmes personnes, la forme du féminin singulier est alle, et non elle, et les variantes du pluriel eusse et eux-autres figurent aux côtés de ils. Il faut noter de plus que la $3^{\mathrm{e}}$ personne du pluriel n'est pas marquée pour le genre, uniquement pour le nombre, comme l'illustre la phrase qui suit.

(1) Quand mes petites filles a commencé à le... ça, ils adoraient leur grand-mère

En ce qui concerne les formes conjointes objets directs et indirects, on observe peu de différences avec les autres variétés de français. Il s'agit principalement la forme de la $3^{\mathrm{e}}$ 
personne lé à l'impératif, non marquée, ni pour le genre, ni pour le nombre comme le montrent les exemple (2) et (3) où lé pronominalise respectivement une pomme et mon accordéon.

(2) Allez mange ! C'est une pomme vert. Goûte-lé, i dit, tu vas voir.

(3) I dit : «Tiens j'vas t'prêter mon accordéon, garde-lé aussi longtemps comme tu veux »

Il faut noter également la quasi-disparition de leur, remplacé par les qui fonctionne aussi bien comme un objet direct qu'indirect ainsi que l'illlustrent les phrases ci-dessous où les est COD en (4) et COI en (5).

(4) J'les connais bien!

(5) Enfin alle a commencé à les parler en anglais, tu connais

Il ne s'agit pas en (5) d'un changement de construction du verbe parler comme le montre la présence de la préposition à devant le NP en (6).

(6) Ça pouvait pas y parler à elle, dire les affaires eusse voulait y dire

\subsubsection{Analyse des pronoms conjoints du français louisianais}

Un examen plus approfondi de l'emploi des formes conjointes du français louisianais mène toutefois à remettre en question la classification comme clitique des formes composées dans leur emploi conjoint. Dans cette section, je montrerai que le fonctionnement de ces formes diffère en plusieurs points de celui des formes traditionnellement considérées comme clitiques, telles que je, tu, il, alle, on et ils et qu'elles ne peuvent être analysées selon les mêmes lignes, ce qui mène à scinder en deux le paradigme des pronoms conjoints de cette variété : les pronoms clitiques et les pronoms faibles, reprenant en cela la terminologie de Cardinaletti et Starke (1994 et 1999).

\subsubsection{Les clitiques}

La première classe de pronoms conjoints, les clitiques, rassemble les formes je, $t u, i l$, alle, on et ils, qui n'ont pas d'équivalent lexical disjoint, la forme disjointe correspondante étant, respectivement, moi, toi, lui, elle, nous-autres et eux-autres ou eusse pour ils. Ce premier groupe de marqueurs est sujet à des phénomènes phonologiques qui ne s'appliquent pas aux formes disjointes. C'est le cas des règles de sandhi externe (restructuration prosodique). La liaison et l'enchaînement s'appliquent systématiquement entre eux et le mot qui les suit :

(7) Ils adoraient leur grand-mère [izadorelørgrãmer]

(8) Dad il a travaillé une grosse grosse journée d'ouvrage [dad ilatravaje]

Ils font l'objet de réductions phonétiques; à titre d'exemple, alle est réduit à [a] et ils est réduit à [i] dans l'énoncé ci-dessous : 
(9) A peut pas manger les deux crabes parce qu'ils sont gros [apøpamãzeledøkrab paskisõgro]

Cette catégorie de formes conjointes constitue, contrairement aux formes disjointes, une unité prosodique avec l'élément lexical adjacent. En (10), par exemple, le locuteur observe une pause entre le DP plein sujet, les Reed, qui forme un groupe intonatif à lui seul, et le syntagme verbal, qui forme un nouveau groupe rythmique avec l'objet direct :

(10) Les Reed avaient tout la propriété

[lerid avetulaproprijete]

En (11) ci-dessous, le pronom disjoint lui, porteur d'un accent contrastif, ne se lie pas au verbe qui le suit et forme une unité prosodique distincte de celle formée par le syntagme verbal :

(11) Et lui connait le nanglais bien

[elui konelønãgle bjẽ]

En (12), en revanche, le sujet conjoint, $i l$, s'incorpore au verbe qui le suit et forme avec lui une unité prosodique :

(12) Et là, euh... il avait une vieille place

[ela $\varnothing \quad$ ilaveønvjcjplas]

La syntaxe de ces marqueurs se distingue, elle aussi, en plusieurs points de celle de leurs pendants disjoints. Ils ne peuvent être ni coordonnés, ni modifiés et ils ne peuvent pas être effacés dans les structures coordonnées, ce qu' 'illustrent les exemples ci-dessous :

(13) * Il et alle va jamais à des bals

(14) * Il seul a venu

(15) J'travaillais en été avec mon beau-père et j'mettais des briques quand j'avais quatorze ans

Les propriétés de ces formes, qui se distinguent des pronoms disjoints aussi bien dans le domaine de la syntaxe que de la phonologie, les rapprochent des clitiques spéciaux de Zwicky (1977), mais leur emploi dans le redoublement du sujet, amplement attesté dans cette variété, y compris celui des sujets quantifiés, comme l'illustrent les exemples ci-dessous, indique qu'ils évoluent vers le statut affixal :

(16) Chacun i fait ça i veut

(17) Un tas de les vieux Cadiens ça disait un graphophone plutôt qu'un phonographe

(18) À la maison, tout le monde ça parle le français

Nous retrouvons ces caractéristiques pour les pronoms conjoints objets même si le redoublement de l'objet, attesté en français louisianais, n'est que sporadique.

Ces pronoms clitiques s'analysent en conséquence comme des faisceaux de traits morphologiques (personne, nombre et genre). Les deux structures arborescentes ci-dessous, la première représentant la structure des DP pleins et des pronoms disjoints, et la seconde, celle 
des clitiques, montrent que ces derniers font état d'un haut degré de déficience morphologique et n'ont conservé que la partie inférieure de la structure morphologique.

Structure des DP-pleins

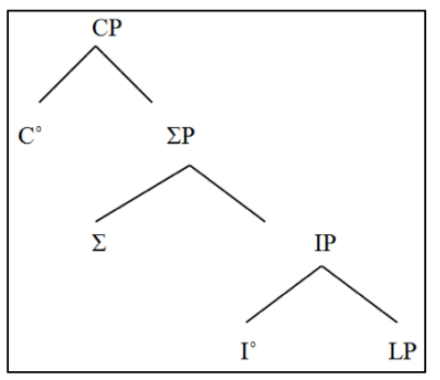

Structure des clitiques

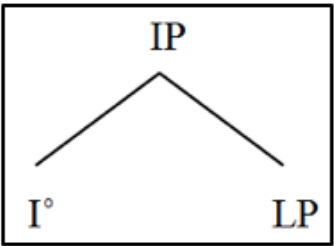

\subsubsection{Les pronoms faibles}

La seconde classe de pronoms conjoints, les pronoms faibles, arborent une même forme lexicale que leurs homologues disjoints, à savoir les formes lui, ça, nous-autres, vousautres, eux-autres/eusse. Leur emploi conjoint est marqué par des phénomènes d'ordre phonologique, tels que l'absence d'accent propre, un contour intonatif plat, leur enchaînement au verbe et le fait qu'ils peuvent faire l'objet d'une réduction phonétique.

En (19), eux-autres est inaccentué et n'est pas séparé du verbe qui le suit par une pause ; il forme avec lui une unité prosodique. On note également la présence d'un accent final de mot sur les infinitifs, emmener et entendre et sur le verbe danse :

(19) Oui ça aide. Et aussi, tu connais ça aide beaucoup avec les enfants parce que asteur euh... euh... le monde peut aller manger dans un restaurant. Eux-autres peut [ø-zot$\mathrm{p} \varnothing]$ emmener les enfants. Les enfants peut apprendre à danser. Eux-autres danse [ $\varnothing-$ zot-dãs] avec leurs enfants, et eux-autres peut entendre la musique [ø-zot pø-ã-tãd-lamy-zik]

En (20), eux-autres s'enchaîne avec le verbe qui le suit :

(20) Eux-autres étaient [ø-zo-te-te] rationned

Le pronom lui apparaît fréquemment dans cet emploi conjoint. La distinction entre ses emplois disjoints et conjoints est exemplifiée ci-dessous ; le premier énoncé présente l'emploi disjoint et le second, conjoint et donc faible :

(21) Ko! Luí connaît jouer

(22) Mais lui a jamais joué rien 
Les copies d'écran de Praat ${ }^{1}$ de ces exemples permettent de visualiser ces différences. La copie d'écran de l'exemple (21) signale un pic sur lui, accentuation liée aux formes disjointes :

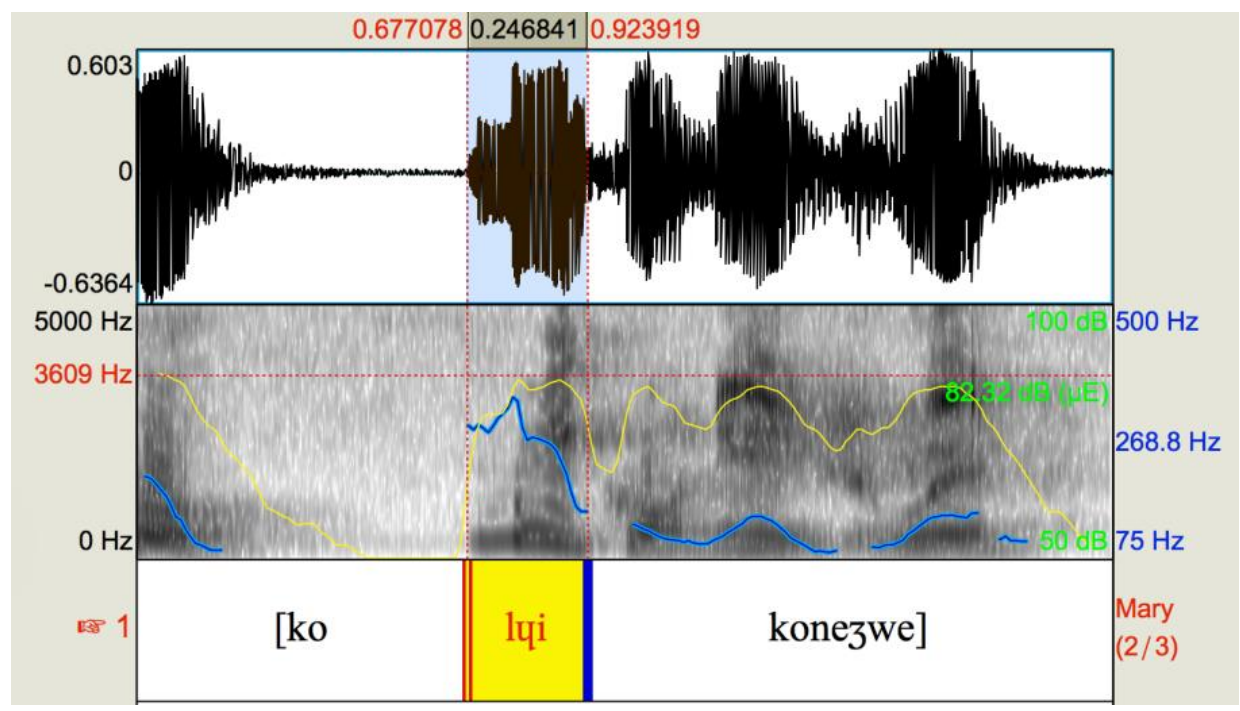

En (22), en revanche, l'intonation plate de la séquence lui a et l'absence de pause entre lui et $a$ montrent que ces deux mots participent d'une même entité prosodique :



La forme toi n'est pas attestée dans un emploi conjoint dans notre corpus. La forme moi a été relevée, une unique fois, dans l'occurrence qui suit, où il n'y a pas de pause entre moi et l'aime qui forment un groupe intonatif :

(23) Moi l'aime manière qu'i joue, lui.

\footnotetext{
${ }^{1}$ Praat est un logiciel de phonétique d'accès libre permettant l'analyse de données sonores, programmé par Paul Boersma et David Weenik de l'Université d'Amsterdam.

Ces copies d'écran de Praat sont présentées ici uniquement pour permettre de visualiser, dans une certaine mesure, le contour mélodique de ces structures, la présence ou non d'un accent final et la présence ou l'absence de pause. Elles ne constituent pas un véritable argument en faveur de l'une ou de l'autre des interprétations du double marquage du sujet en cadien, ce qui requerrait une analyse fine de plusieurs paires d'exemples enregistrés dans de meilleures conditions, les bruits ambiants étant susceptibles d'interférer.
} 
Le pronom ça est fréquemment attesté dans cet emploi, ce qu'illustre l'exemple cidessous où ça reprend le sujet disloqué ce monde.

(24) Parce que ce monde, ça dépense pas d'argent.

Le fonctionnement de cette seconde série de pronoms, qui représentent une variante phonologique d'une forme disjointe, mène à les analyser plus comme des clitiques simples dans le sens de Zwicky (1977). Ils se distinguent en effet, à la fois des pronoms disjoints et des clitiques, décrits respectivement en 4.2 et en 4.3.2.1. À la différence de leurs homologues disjoints, ils s'attachent au verbe dont ils sont l'argument et s'enchaînent avec lui. Ils peuvent faire l'objet de réductions phonétiques et leur distribution est plus réduite. Ils se distinguent également de la classe des pronoms sujets conjoints clitiques en ce qu'ils ne peuvent s'employer dans les redoublements de sujets, mais s'utilisent, en revanche, pour reprendre un sujet disloqué. Ce sont des têtes lexicales avec une structure arborescente intermédiaire entre celle des pronoms disjoints et celle des clitiques. L'arbre ci-dessous montre que ces pronoms ont une structure morphologique plus complexe que celle des pronoms clitiques et que leur degré de déficience est moins élevé :

Structure des pronoms faibles

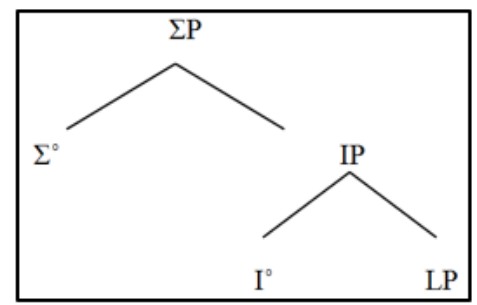

\subsubsection{Les marqueurs pronominaux objets}

Les marqueurs objets conjoints du français louisianais se divisent eux aussi en deux classes constituées, l'une par les objets proclitiques, et l'autre, par les objets enclitiques.

Les proclitiques objets me, te, le, la, les, y, nous, vous, se n'ont pas d'équivalent lexical disjoint, le paradigme disjoint correspondant étant moi, toi, lui, elle, nous-autres, vousautres, eux-autres. Leur fonctionnement, qui arbore plusieurs points communs avec celui des marqueurs sujets conjoints du premier groupe, mène à les analyser eux aussi comme des clitiques. Ils peuvent faire l'objet de réductions phonétiques et ils se lient au mot verbal qui les suit pour former avec lui une entité prosodique comme l'illustre l'exemple ci-dessous :

(25) Pop Gilbert, tu t'rappelles Pop Gilbert?

$$
\text { [tytrapel popzilber] }
$$

Leur position par rapport à leur hôte est fixe, à la gauche du verbe, et diffère de celle de leurs homologues disjoints qui se placent après le verbe, ainsi que le montre l'énoncé en (26) :

(26) J'veux tu m'dis à moi quoi ce qui les fait rire.

[3vø tymdiamwa kwaskileferir] 
L'énoncé en (26) illustre de plus le redoublement possible de l'objet dans cette variété, même s'il est plus rarement attesté que celui du sujet.

Le second groupe de pronoms objets conjoints, les enclitiques objets, moi, toi, lé, lui, nous, vous, arborent, à l'exception de lé, une forme identique à la variante disjointe et se rapprochent en cela des pronoms faibles. Leur emploi est toutefois extrêmement restreint et se limite aux constructions impératives, aussi bien positives que négatives :

(27) J'dis : « Ouais, va t’en. Amuse-toi ! "

(28) Tracasse-toi pas.

(29) Il a dit : «Mange ! C'est une pomme vert. Ben, goûte-lé, i dit, tu vas voir. »

Leur place est fixe, immédiatement à la droite de leur hôte, le verbe à l'impératif. Contrairement à leurs homologues disjoints, ils ne peuvent porter d'accent contrastif. Ils sont certes susceptibles de porter un accent, comme c'est le cas de toi en (28), par exemple, mais il s'agit d'un autre type d'accent, d'un accent final de groupe prosodique. Sur le plan syntaxique, ils ne peuvent être ni modifiés, ni coordonnés.

Bien que ces formes ne fassent pas l'objet d'une réduction phonétique, nous les analysons comme des pronoms faibles, des clitiques simples, car elles sont rattachées à un hôte, le verbe, et leur distribution est réduite.

\section{Conclusion : Trois classes de pronoms en français louisianais}

Le français louisianais de distingue des autres variétés de français en ce que son système pronominal s'organise en trois paradigmes, en trois classes de pronoms qui se situent sur un continuum du type :

pronom disjoint $\rightarrow$ pronom faible $\rightarrow$ clitique $\rightarrow$ affixe marqueur d'accord $\rightarrow$ zéro

Leur analyse montre que la distinction entre ces classes est basée sur le degré de déficience structurelle des membres des différentes classes :

Les pronoms clitiques : faisceau de traits (affixes)

Les pronoms faibles : Têtes lexicales $(\mathrm{X})$

Les pronoms disjoints : syntagmes déterminatifs (XP)

Leur analyse a également montré que la distinction entre pronoms disjoints et pronoms faibles relève de la syntaxe et de la phonologie, alors que la distinction entre pronoms faibles et clitiques ressort à la fois de la morphosyntaxe et de la phonologie.

Les paradigmes des pronoms du français louisianais auxquels l'analyse permet d'aboutir sont donc de la forme ci-dessous : 


\begin{tabular}{|c|c|c|c|c|}
\hline \multirow{2}{*}{ Personnes } & \multicolumn{2}{|c|}{ Clitiques } & \multirow{2}{*}{ Pronoms faibles } & Pronoms disjoints \\
\cline { 2 - 3 } & Sujet & Objet & moi & moi \\
\hline 1ssg. & je & me & & toi \\
\hline 2ssg. & tu & te & & lui, elle, ça \\
\hline 3ssg. & vous & vous & & nous-autres \\
\hline 1pl. alle, ça & le, la, y, lui, se & lui, elle, lé, ça & vous-autres \\
\hline 2pl. & on & nous, se & nous-autres & eux-autres, eusse, ça \\
\hline 3: pl. & ça & vous, se & vous-autres, ça & \\
\hline
\end{tabular}

Le français louisianais se distingue ainsi des autres FPI par l'émergence d'une nouvelle classe de pronoms conjoints, les pronoms faibles, qui vient combler le vide laissé par la disparition de certains clitiques du paradigme et lui évite de devenir une langue à sujet nul.

\section{Bibliographie}

Auger, J. 1995. «Les clitiques pronominaux en français parlé informel : une approche morphologique ». Revue québécoise de linguistique. Volume 24, numéro 1, 21-60.

Auger, J. 2003. «Les pronoms clitiques sujets en picard : une analyse au confluent de la phonologie, de la morphologie et de la syntaxe ». Journal of French Language Studies. Volume 13, numéro 1, 1-22.

Cardinaletti, A. et Starke, M. 1994. «The Typology of Structural Deficiency. On the Three Grammatical Classes ». University of Venice Working Papers in Linguistics. Numéro 4, 41-109.

Cardinaletti, A. et Starke, M. 1999. « The Typology of Structural Deficiency » in H.van Riemsdisjk (éd.), Clitics in the language of Europe. Language Typology. Volume 8. 145-233. Berlin : Mouton.

Gelderen, Elly van. 2011. The Linguistic Cycle: Language Change and the Language Faculty. Oxford : Oxford University Press.

Girard, F. 2012. «Quelle analyse pour les pronoms clitiques du cadien ?» in F. Neveu, V. Muni Tokr, P. Blumenthal, T. Klingler, P. Ligas, S. Prévost et S. Teston-Bonnard (éds.), Actes du 3e Congrès mondial de linguistique française. 2351-2365. Paris : EDP Sciences. 2351-2365.

Girard, F. 2013. « Pronominal markers in Cajun French » in C. M. Salvesen et Helland, H. P. (éds), Challenging Clitics. 187-208. Amsterdam. John Benjamins Publishing Company. 
Girard, F. 2014. « L'expansion de ça en cadien » in H. P. Helland et C. M. Salvesen (éds.), Affaire(s) de grammaire. 205-217. Oslo : Novus Forlag.

Girard Lomheim, F. 2016. L'expression de la personne dans un français louisianais. Grammaire du système pronominal cadien des paroisses de Saint-Landry et Vermilion. Thèse de doctorat, Université d'Oslo.

Girard Lomheim, F. 2017. Le pronom dans le français de Louisiane. Trois siècles de cheminement. Paris : L'Harmattan.

Kayne, R. 1975. French Syntax : The transformational cycle. Cambridge MA : The MIT Press.

Kayne, R. 1983. « Chaînes, catégories extérieures à S et inversion complexe en français ». Langue française. Numéro 58, 36-65.

Rizzi, L. 1986. «On the Status of Subject Clitics in Romance » in O. Jaeggli et C. SilvaCorvalán (éds.), Studies in Romance Linguistics. 391-419. Dordrecht : Foris Publications.

Roberge, Y. 1990. The syntactic recoverability of null arguments. Montréal : McGill-Queen's University Press.

Zribi-Hertz, A. 1994. «La syntaxe des clitiques nominatifs en français standard et avancé. » Travaux de linguistique et de philologie. Volume 32, 131-147.

Zwicky, A. 1977. On Clitics. Bloomington IN : Indiana University Linguistics Club. 